\title{
Reúso pragmático de neumáticos en un proyecto educativo para alcanzar competencias del desarrollo sostenible
}

\author{
JÉSSICA Guevara SÁEnZ de Viteri \\ FÉlix TINOCO ÁNGELES ${ }^{2}$ \\ EmÉrita Delgado Plaza ${ }^{3}$ \\ JuAN CALDERÓN Cisneros ${ }^{4}$
}

Recibido: 12/09/2020 Aceptado: 07/12/2020 Publicado: 31/12/2020

\begin{abstract}
RESUMEN
Aplica filosofía pragmática combinando Competencias de Objetivos de Desarrollo Sostenible (ODS): Autoconciencia del valor ambiental con el fin de aportar con formas de reúso de Neumáticos Fuera de Uso (NFU) integrados al trabajo colaborativo en Proyecto Educativo Institucional (PEI) Tierra de Niños y Niñas y Adolescentes (TINI); en unidades educativas de nivel medio en Ecuador contribuyendo con mejoramiento del entorno. El Programa Reúsa Llanta del Ministerio de Industrias y Productividad Ecuador, estimó que 300 mil llantas son destinadas a botaderos $u$ otros sitios y a partir de la ejecución del TINI se recuperó aprox el $2 \%$ sirviendo al ornamento de las unidades sujeto de investigación. Se aplicó prueba de Bartlett cuya significancia es $(P<0,01)$, lo que permitió rechazar la hipótesis nula de que la matriz de correlaciones es igual a la matriz identidad, y se concluye que efectivamente existe asociación entre las variables originales y una reducción dimensional de las actividades del proyecto educativo de reúso pragmático de neumáticos usados considerados como desechos especiales en el Ecuador. El pragmatismo ambiental da lugar a la formación de multiplicadores logrando cambios significativos en hábito de reusar los desechos especiales, así como el fomento de trabajo colaborativo y estímulo de creatividad; alcanzando la concienciación ambiental.
\end{abstract}

Palabras clave: Reúso; pragmatismo ambiental; prueba de Bartlett; desarrollo sostenible; desechos especiales.

Pragmatic tire ReUSe in an EDUCaTIONAL PROJECT TO ACHIEVE SUSTAINABLE DEVELOPMENT SKILLS

\section{ABSTRACT}

Applies philosophical pragmatism current combining Sustainable Development Objectives Competences: Selfawareness of environmental value in order to contribute with NFU Reuse forms integrated to collaborative work in (PEI) Institutional Educational Project Land of Children and Adolescents (TINI); as a curricular requirement in medium-level educational units in Ecuador contributing to the improvement of the environment. The Tire Reuse Program of the Ministry of Industries and Productivity Ecuador, estimated that 300 thousand tires are destined to dumps or other sites and after the execution of the TINI recovered approximately $2 \%$ serving the ornament of the units under investigation. Bartlett's test was applied, whose significance is $(P<0.01)$, which allowed rejecting the null hypothesis that the correlation matrix is equal to the identity matrix, and it is concluded that there is indeed an association between the original variables and a reduction dimensional activities of the educational project

\section{INTRODUCCIÓN}

El Desarrollo Sostenible involucra modelos de comportamiento integradores que reduzca la generación de desechos sólidos con la mejor alternativa de aprovechamiento de los neumáticos fuera de uso NFU evitando; abandono, quema y/o destinos inadecuados en vertederos, quebradas y ríos. De acuerdo a Joas (1998) indica que el concepto de sostenibilidad proviene de la constatación de la limitación de los recursos naturales apuntando el funcionamiento de la sociedad industrial al análisis y reducción de los efectos del consumismo que ponen en peligro la supervivencia del planeta a largo plazo.

Esta interpretación conceptual del desarrollo sostenible implica la relación futura de los humanos con el entorno urbano, debido a que el número de personas que habitan en urbes excede al número de habitantes en áreas rurales; y actualmente se mantienen discusiones sobre desarrollo urbano sostenible y su tendencia a centrarse en economía versus conservación ambiental, resaltando el papel esencial de las ciudades como hábitat humano postulando cambios sociales pacíficos y graduales, así como generación de propuestas organizadas y planificadas que modifican la relación con la naturaleza y con la sociedad. Ramírez, Sánchez y García (2003). De esta manera, el desarrollo sostenible ha creado una gran adhesión porque ofrece puntos centrales de consenso a nivel mundial.

Esta óptica, afirma la complementariedad de los objetivos de desarrollo sostenible con metas alcanzables para cada nación

1 Ingeniera Industrial- Escuela Superior Politécnica del Litoral mención en Manejo de Efluentes Industriales, Magister en Ingeniería Ambiental, docente de Universidad de Guayaquil - Facultad de Filosofía, Letras y Ciencias de la Educación, Guayaquil, Ecuador.

ORCID: https://orcid.org/0000-0003-1148-0014

E-mail: jessica.guevaras@ug.edu.ec

2 Magíster en Gestión de Operaciones y Servicios Logísticos por la Universidad Nacional Mayor de San Marcos (Lima, Perú). Consultor independiente.

ORCID: https://orcid.org/0000-0002-2630-4657 E-mail: tinocofelix@gmail.com

3 Ingeniera Mecánica- Escuela Superior Politécnica del Litoral; Master y Doctorado en Energía Renovable y Sostenibilidad Energética. De la Universidad Santiago de Compostela, España, Docente en Facultad de Ingeniería en Mecánica y Ciencias de la Producción ESPOL, Guayaquil, Ecuador. ORCID: https://orcid.org/0000-0003-1647-852X

E-mail: eadelgad@espol.edu.ec

4 Ingeniero en Estadística -Informática- Escuela Superior Politécnica del Litoral; Docente Titular de la Facultad de Salud en la Universidad Nacional Estatal de Milagro, Milagro, Ecuador, Investigador acreditado por Senescyt-Reg-Inv:18-02756.

ORCID: https://orcid.org/0000-0002-8167-8694

E-mail: jcalderonc@unemi.edu.ec 
of pragmatic reuse of used tires, considered as special waste in Ecuador. Environmental pragmatism gives rise to the formation of multipliers achieving significant changes in the habit of reusing special waste, as well as the promotion of collaborative work and stimulation of creativity; achieving environmental awareness.

Keywords: Reuse; environmental pragmatism; Bartlett test; sustainable development; special waste. de forma multidimensional aplicado a más de un problema en varios sectores, Salazar (2012) consecuentemente se plantea el término sostenible para las aplicaciones globales del reúso de los NFU como parte de la solución de un problema nacional del Ecuador en la Provincia del Guayas, ciudad de Guayaquil.

Tal como indican Ramírez et al. (2003) el tema del desarrollo sustentable promueve cambios graduales en los niveles sociales; introduciendo el Proyecto Educativo Integral PEI -TINI como parte del currículum en colegios para adecuar, conservar y mantener áreas verdes que contribuyan al mejoramiento del entorno educativo, desarrollo de destrezas y valoración ambiental a través de las actividades planteadas por docentes en periodos anuales Flavell (1979). Se enlaza esta nueva visión del concepto como el reflejo práctico para la valoración del entorno dando cumplimiento al requerimiento educativo en todas las instituciones educativas del Ecuador, incorporando soluciones como el reúso de los NFU.

Este artículo es producto de un proyecto fundamentado en la filosofía pragmática, combinando el concepto de las Competencias de Objetivos de Desarrollo Sostenible (ODS) cuyo fin aporta a la gestión ambiental de desechos especiales como es el caso de los Neumáticos Fuera de Uso (NFU) presentes en el entorno de las instituciones educativas de la ciudad de Guayaquil, Distrito 09008, Pascuales ubicadas en la Provincia del Guayas del Ecuador; así como formas de reúso. Se integran elementos como el trabajo colaborativo en las actividades del Proyecto Educativo Institucional (PEI) Tierra de Niños y Niñas y Adolescentes (TINI); contribuyendo con el mejoramiento del entorno a través de su metodología.

Para el análisis estadístico de datos se plantea la prueba Chi Cuadrado; complementando con el análisis factorial exploratorio Prueba de $\mathrm{KMO}-B$ artlett.

Siendo la hipótesis nula $\left(\mathrm{H}_{0}\right)$ No es posible agrupar las actividades del Reuso pragmático de neumáticos en tres competencias de desarrollo sostenible: análisis crítico, reflexión sistemática y toma de decisión con responsabilidad : mientras que la hipótesis $H_{1}$ :Es posible agrupar las actividades del Reuso pragmático de neumáticos en tres competencias de desarrollo sostenible: análisis crítico, reflexión sistemática y toma de decisión con responsabilidad.

\section{Importancia de la reutilización de los neumáti- cos fuera de uso}

A nivel mundial, los neumáticos fuera de uso representan un problema ya que se trata de la gestión de desechos sólidos especiales y evidentes efectos negativos ambientales por incorrecta disposición final. Este último término aplicado por la Legislación Ambiental del Ecuador para referirse a los neumáticos desechados.

La problemática de la disposición final de los neumáticos fuera de Uso (NFU), hace que los gobiernos a nivel mundial busquen implementar alternativas en conjunto con regulaciones orientadas a la disminución de generación del residuo. En el caso de Ecuador durante el año 2018 se planteó un incentivo industrial denominado Sistema Ecuatoriano de Gestión Integral de neumáticos usados (SEGINUS), contando con la participación del Ministerio de Ambiente logrando cubrir el $80 \%$ del mercado comercializador de neumáticos; cobrándose un ecovalor reflejado en las facturas de los usuarios al momento de comprar los neumáticos nuevos. Un dato relevante es que el $90 \%$ del mercado de importación de neumáticos corresponde para vehículos livianos según Reporte de Ecuador en Cifras INEC.

Adicionalmente, se reportó la cantidad de $2.4 \mathrm{mi}-$ llones de neumáticos desechados durante el año 2018 debido al crecimiento del parque automotor en la Provincia del Guayas en Ecuador. Según el INEC (2015), por otro lado el Reporte Ecuador en cifras registró 1'925.368 de vehículos correspondiente al $57 \%$ más que en el 2010.

Los usuarios refirieron que no saben qué hacer con este desecho debido a que no los pueden vender y en pocos casos son aceptados por las casas comercializadores. Volviéndose imperiosa la necesidad de emprender soluciones creativas no sólo a nivel nacional sino local, involucrando acciones institucionales y para el presente caso se trata de un proyecto que impulsa el aprendizaje colaborativo bajo enfoque pragmático ambiental a nivel de instituciones educativas de nivel medio.

De manera tal, que el incremento de NFU es considerable y la búsqueda de alternativas para su aprovechamiento es necesaria; sin provocar daños 
ambientales por la eliminación de volúmenes de desechos y/o emisiones.

En respuesta a esta necesidad, se realizó un proyecto innovador educativo ambiental con enfoque a los procesos de selección y reúso de los neumáticos fuera de uso NFU aplicado al diseño de modelos decorativos en instituciones educativas que promueven el reúso pragmático de los neumáticos usados basado en tres dimensiones: Análisis Crítico, Reflexión sistémica y toma de decisiones colaborativas con sentido de responsabilidad hacia generaciones futuras; dimensiones consideradas como competencias para la sostenibilidad de acuerdo a la UNESCO.

Se analizará estadísticamente a través de la Prueba Chi Cuadrado la Hipòtesis de Incidencia de actividades del Reuso pragmático de neumáticos para desarrollar competencias de desarrollo sostenible, así como se aplicará la prueba KMO y Barlett, en la agrupación de actividades tales como: selección de formas, tamaños de Neumáticos usados, colores, funcionalidad, evaluación y colaboración entre grupos, creatividad y comparación de diseño, fomento de autoconciencia por efectos socio-ambiental, efectos sanitarios por inadecuado manejo de neumáticos usados, aportaciones de aplicación del concepto de reúso; de acuerdo a las tres dimensiones anteriormente detalladas.

\section{COMPETENCIAS PRAGMÁTICAS DE OB- JETIVOS DE DESARROLLO SOSTENIBLE COMO BASE DEL TRABAJO COLABORA- TIVO Y CREATIVO}

De acuerdo a Tünnerman y Souza (2003) el concepto de sostenibilidad integra la complejidad de aspectos sociales, económicos y ecológicos, lo que implica la relación entre: personas de diferentes culturas que puedan convivir con equidad de derechos y deberes; en un sistema económico de naciones que permita la distribución justa de la riqueza, para cubrir necesidades básicas optimizando el uso de los recursos, y la práctica del valor del respeto hacia todas las formas de vida incluyendo los ciclos naturales.

Para incorporar las competencias transversales de los objetivos de desarrollo sostenibles en cualquier realidad, estudio, actividad profesional y/o docente, hay que encontrar el punto confluente, e integracional entre la dimensión económica, dimensión ecológica y la dimensión social. Bambrick (2011) en la integración de la dimensión social está la vertiente de la corriente filosófica pragmática que enfoca el punto de vista naturalista.
Friedrich Nietzsche postuló conceptos naturalistas y voluntaristas del ser humano, citó Rolston III (2004) en que la verdad no es un valor teórico, sino una expresión para designar la utilidad en función del juicio que conserva la vida y sirve a la voluntad de poder.

Se plantea la incorporación del conocimiento del reúso de desechos sólidos y su aplicabilidad en los ejes transversales: elementos ecológicos, cuidado ambiental, cuidado a la salud que alcanzan la reducción de riesgos y peligros a la salud de pobladores que están expuestos a desechos contaminantes. Estos elementos están inmersos en la presente investigación como efectos importantes que justifican el desarrollo de la investigación.

Así mismo,Weston (1985) indica que el pragmatismo ambiental por principio elabora autoevaluaciones acerca de la relación existente entre el mundo natural y las actividades antropogénicas; para este caso la inclusión de actividades complementarias curriculares en las unidades educativas de nivel medio que infieren el valor de la especie, canalizando acciones hacia el mundo natural que está más allá de lo que el ser humano puede comprender, denominando racionalismo a la posición epistemológica que ve en el pensamiento; esto es, la fuente principal del conocimiento humano. Los juicios fundados en el pensamiento procedentes de la razón, poseen necesidad lógica y validez universal; los demás no.

Encuadrándose durante todo el análisis de la selección de alternativas del reúso de los NFU a través de la implementación de: camineras, maceteros, figuras decorativas, asientos, pisos para gimnasia, columpios y separadores de áreas verdes.

Murillo (2004) aporta que Kant escribe en la Introducción a su Crítica de la razón pura: «No hay duda alguna de que todo nuestro conocimiento comienza con la experiencia [...] por lo tanto, todo lo que se propone realizar es producto de su condición tomando en cuenta la verdadera dimensión; y a la gestión del conocimiento como elemento de tipo integrador.

Los autores de esta investigación lo identifican como modelo pragmático que muestra al ser vivo desde la perspectiva multidimensional incluyendo la conciencia del cuidado del entorno que se ve afectado por el aumento de desechos sólidos peligrosos como lo son los NFU sin darle opciones claras de valor agregado dentro de la cadena productiva local; siendo este un enfoque holístico que permite construir conocimientos a través de experiencias 
significativas durante el acto del desarrollo humano; transformando con conocimiento las vías del aprendizaje significativo a favor del entorno y del aprovechamiento de residuos en un contexto que puede estratégicamente incorporarse a la realidad de las unidades educativas empleando emprendimientos creativos y eficaces que promuevan la solución de problemáticas ambientales.

La aplicación de las competencias transversales de los Objetivos de Desarrollo Sostenible (ODS) está basado en dar soluciones a los problemas asociados al respeto y cuidado del medioambiente; así como al trabajo colaborativo que puede desarrollarse en componentes ambientales, indistintamente y que el ser humano puede combinar actividades dentro de lo ético-ambiental para la resolución de problemas que alcancen la reducción de impactos ambientales de manera significativa. Situaciones que en las unidades educativas van alineadas al Proyecto Educativo Integral PEI -TINI.

Para los teóricos de la corriente pragmática, no cabe el absolutismo como factor determinante al valor intrínseco; la función primordial del pensamiento es promover acciones que fomenten la complacencia con el entorno en escenarios concretos cuya finalidad será propugnar acciones antropogénicas en contextos específicos en que la verdad emerge a partir del hombre con la práctica.

El objetivo principal del pragmatismo ambiental es alcanzar permutaciones en individuos y políticas ambientales a nivel global, fundamentado en que todo ser natural tiene valor intrínseco con consideración moral. Para la ejecución del Proyecto Educativo Integral PEI -TINI se planteó el reúso de los NFU, empleando competencias de: reflexión sistémica con autoconciencia, toma de decisiones responsables resolviendo problemas, Análisis Crítico para el planteamiento de actividades

\section{LA RELACIÓN DEL TRABAJO COLABO- RATIVO, APRENDIZAJE SIGNIFICATIVO CON COMPETENCIAS DE LOS ODS}

El trabajo colaborativo es una de las competencias transversales planteadas en los ODS que tiene como finalidad compartir conocimientos para construir conocimiento común con el único interés de promover la mejora en los resultados de cualquier tipo de situación para que a la postre puedan generalizarse.

El docente busca el aprendizaje significativo dentro del contexto estratégico curricular para su posterior interpretación- práctica cuyas características son: actividad, efectividad, y colaboración a partir de la información obtenida del medio; fomentando destrezas y habilidades en estudiantes, así como criticidad. Se optimiza la capacidad comparativa usando criterios de clasificación desde un punto de vista reflexivo que desarrolla la Competencia de Reflexión Sistémica con Autoconciencia según los ODS.

Se plantea como estrategia el fomento del trabajo colaborativo entre docentes, integrando el desarrollo en estudiantes y el aprendizaje profesional de los profesores de acuerdo a lo propuesto por Collazos y Mendoza (2006); a partir del reconocimiento del trabajo colaborativo como instrumento fundamental. Para lograr esto, se citan cinco aspectos claves: Confianza, aprendizaje de los estudiantes, manejabilidad, apoyo y privacidad.

Teniendo en cuenta estos aspectos, se indica que el trabajo colaborativo fomenta la creatividad durante la práctica, y cada participante emite ideas que en conjunto dan como resultado nuevos productos aplicables a los requerimientos curriculares; alcanzado como fin último la resolución de los problemas bajo los estándares educativos que es otra de las competencias transversales de los ODS según el Instituto Latinoamericano y del Caribe de Planificación Económica y Social (2000). De acuerdo a los aportes de Cozar (2001), se afirma que existe un fuerte vínculo entre la corriente pragmática, creatividad y trabajo colaborativo; influyendo de manera determinante en el aprendizaje significativo.

\section{METODOLOGÍA DEL PEI TINI Y ALTERNA- TIVAS DE REÚSO DE LOS NFU}

EI Proyecto Educativo Integral PEI -TINI es una metodología ambiental de origen peruano que significa Tierra de niñas, niños y jóvenes para el buen vivir y que ha sido asumida en países como Ecuador, entre otros, planteando y organizando actividades cuyo contenido favorezcan a la promoción del amor a la naturaleza y cuidado del entorno. Se busca promover la conciencia ambiental y dentro de este contexto el Ministerio de Educación del Ecuador resolvió la implementación del Proyecto Educativo Integral PEI-TINI mediante acuerdo ministerial Nro. MINEDUC-MINEDUC- ME-2017-00094.

A partir del año 2017, las unidades educativas articulan en su currículo académico el proyecto innovador y que a la fecha su implementación ha reflejado éxito en las diferentes unidades educativas ecuatoriana; relacionando de manera intrínseca la experiencia y practicidad de los educandos a través de su participación en el cuidado medioambiental. 
El proyecto PEI TINI estimula el desarrollo de las competencias transversales de los Objetivos de Desarrollo Sostenible, bajo el enfoque pragmático ambiental de la construcción del conocimiento durante la experiencia significativa del manejo y aprovechamiento de los residuos sólidos que se generan por la cobertura de necesidades antropogénicas; haciendo más significativo el acto de vivir y las aportaciones al desarrollo sostenible; restructurando el conocimiento adquirido a favor del cuidado del entorno y adaptándolo a la realidad local con otras alternativas. Joaquín Leguía, autor de la metodología TINI (Tierra de Niñas, Niños y Jóvenes), expuso el concepto estratégico, de incentivar la investigación, así como el amor por la naturaleza a través de un mayor contacto con esta. Sin embargo, este concepto estratégico ha venido mestizándose según la realidad nacional en cada país, sin perder el propósito del cuidado de la naturaleza.

\section{La metodología TINI consiste en:}

1. Entregar a estudiantes un área en la unidad educativa cuyas dimensiones pueden variar.

2. Planificación de tareas para trabajar en el cuidado y ornamento del área a su cargo.

3. Las actividades implementadas se realizan según las necesidades de la zona donde se ubica la unidad educativa. Las necesidades identificadas pueden corresponder: tipo de producción del sector, siembra de flores y cultivo de ciclo corto, así mismo implica el aprovechamiento de recursos del medio y en este caso se presentan los desechos cuyo concepto se transforma en residuo.
4. Bajo la dirección del docente se realizan las actividades programadas, y ornamentos a elaborarse a partir de materiales encontrados en el entorno. Para el caso expuesto en el presente paper, se refiere a la recolección de Neumáticos Fuera de Uso (NFU) los cuales son cortados, rellenados y/o decorados tomando nuevas formas, funciones y figuras.

5. Los resultados del PEI TINI enfatizan en los estudiantes la importancia de la naturaleza y la biodiversidad existente como parte del entorno, creando un vínculo de protección hacia el medio ambiente y todas sus formas de vida.

La metodología es combinable por la sinergia producida entre la aplicación de los conceptos vinculados tal como está en la figura 1, dado que se analiza la potencial participación de docentes con sus estudiantes en función del aprovechamiento de recursos disponibles en la zona, calificándolos como participación positiva en relación al manejo y gestión del desecho; a través del incremento de áreas verdes en espacios institucionales Gualdrón (2007).

El desarrollo de las competencias ODS como es el caso de autoconciencia ambiental, se produce durante la ejecución de actividades que reflejan el pragmatismo ambiental que guardan estrecha relación con el incremento del conocimiento del entorno que sustenta al ser humano. A su vez, esto se convierte en una variable asociable a la mejora de calidad de vida basada en el aprendizaje significativo sobre el cuidado del entorno sin generar su degradación. Dourojeanni (2000); integrándose a los procesos de gestión para el desarrollo sustentable.

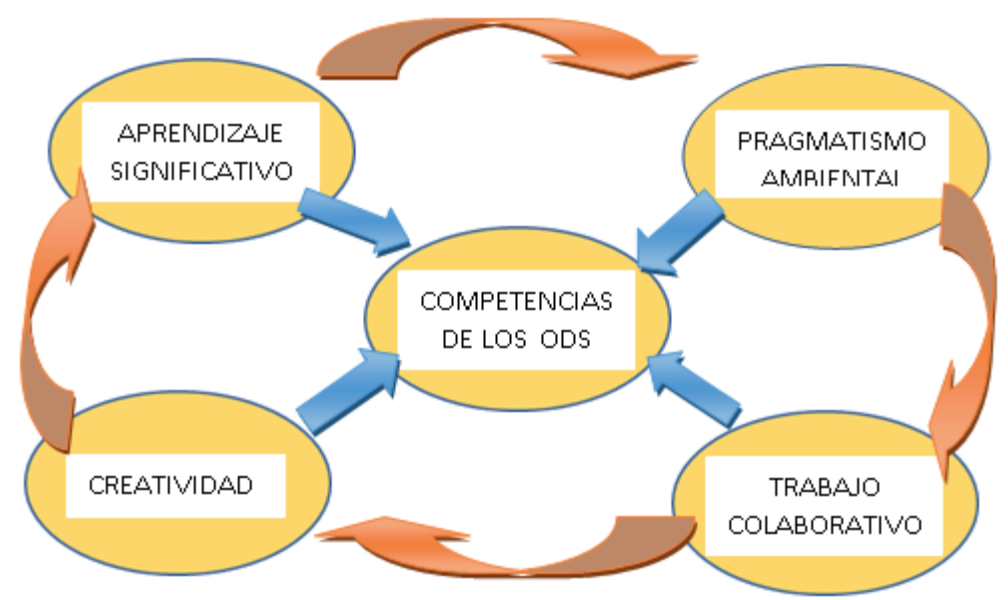

Figura 1. Esquema de relación conceptual vinculante Fuente: Propia, elaborada por autores 2019 
Los temas como: creatividad y trabajo colaborativo se convierten en transversales que se aplican a todas las actividades que se plantean durante las fases del proceso para elaboración de modelos decorativos reusando los NFU.

\section{Fases del Proyecto Educativo Ambiental Reu- sando Neumáticos Usados}

El proyecto constó de cinco fases principales que consisten en:

FASE 1: Análisis de Problemática y Formación de los equipos de trabajo, a partir de observaciones realizadas en el entorno de la unidad educativa, se identificaron lugares donde se encontraban los neumáticos usados y los efectos socio-ambientales, sanitarios que generaban a la comunidad por su mala disposición.

Efectuándose inmediatamente reuniones organizadas por Técnicos especializados en temas socio-ambientales, sanitarios con el propósito de presentar las afectaciones al entorno. Para ello se contó con la participación activa de directivos, docentes de la unidad educativa, padres de familia y se debatieron alternativas con posibles aportaciones enfocadas al reúso.

Se designaron tareas de acuerdo con las habilidades percibidas en los participantes del grupo, definiéndose tareas basadas en las metas propuestas del proyecto y agrupación de actividades que tributaron a la competencia de Desarrollo sostenible: Análisis Crítico.

FASE 2: Selección de materia prima, en la que se procede a la búsqueda de los NFU existentes en la zona previa observación del estado en que se encuentren con el objeto de prevenir accidentes como cortes, caídas ó infecciones debido de la presencia de vectores infectocontagiosos. Los grupos notifican a los docentes acerca de estos casos para solicitar equipos de protección y/o herramientas.

Paralelamente otros participantes del grupo se encargan de la preparación de materiales complementarios tales como: pinturas, brochas, mascarillas, pinturas en spray, diluyente, cartones, hojas para sierras, tijeras metálicas tipo cizallas, entre otros. Así como la preparación y despeje del lugar en el cual se ejecutará la limpieza del neumático usado.

FASE 3: Procesamiento, que implica la limpieza y corte del neumático fuera de uso en caso de necesitarlo, debido a que pudieran encontrarse neumáticos incompletos $\mathrm{o}$ fragmentos deteriorados inutilizables.
Por otra parte, los docentes revisan los avances del listado de actividades asignadas a cada grupo, así como el porcentaje de cumplimiento de acuerdo a los tiempos planteados para el proyecto.

FASE 4: Selección de Diseños Decorativos, durante esta fase existieron reuniones entre los docentes guías y los grupos de trabajo para someter a libre elección la selección del diseño basados en: Número de neumáticos usados encontrados, Número de neumáticos usados íntegros, número de fragmentos de neumáticos, colores a emplear, funcionalidad del diseño y facilidad de realización de cortes debido a la presencia del alma metálica en el interior de la carcaza del neumático usado. Estas actividades tributaron a la competencia denominada Toma de decisiones colaborativas con sentido de responsabilidad hacia generaciones futuras.

Los diseños decorativos seleccionados en base a las condiciones mencionadas fueron:

- Separadores en jardines

- Bases para maceteros

- Divisores de caminería

- Figuras colgantes porta macetas

- Figuras decorativas

Con respecto a los costos asociados en la elaboración de diseños decorativos, es importante resaltar que se incluyen materiales complementarios residuales existentes en las unidades educativas, así como en los domicilios de los estudiantes participantes durante actividades grupales; por lo que se incurre en un costo mínimo que puede variar a partir de 5 usd por diseño aplicable a cada neumático fuera de uso recolectado correspondiente a la compra de detergente por limpieza y pintura de látex ( en caso de no disponer de sobrantes).

A continuación se enlistan los materiales complementarios generalmente empleados: pinturas de látex, fomix, alambres, botellas plásticas vacias, acuarelas y/o temperas, detergente para limpieza, brochas.

En el caso de figuras colgantes porta macetas se suele incorporar bases plàsticas que sirven como contenedor interno para tierra de sembrado. Las llantas aro número 26,20 y 24 de nylon tipo bicicleta se suelen emplear para elaboración figuras decorativas colgantes.

Por otro lado, las llantas aro número 14, 15 y 17 provenientes de autos y motos; son destinadas a las fabricaciones de figuras decorativas, separadores en 
jardines, divisores de caminerìa las cuales deben cortarse con ayuda de docentes y padres de familia.

Mediante la ejecución del proyecto educativo ambiental PEI TINI no sòlo se busca fomentar la implementación y cuidado de áreas verdes, sino acoplar el análisis del reuso pragmático de neumáticos fuera de uso en las diferentes fases enfocado a la elaboración de diseños que incorporen materiales complementarios residuales existentes.

FASE 5: Evaluación grupal, conformada por las siguientes actividades:

- Incremento de interacciones personales

- Conformación de subgrupos supervisados por docentes

- Desarrollo de creatividad y comparación entre opciones de diseño decorativo.

- Integración de conceptos de seguridad y valoración de la relación individual con el entorno basado en experiencias

Las cuales aportan no sólo al trabajo colaborativo, sino que se vinculan con la competencia de reflexión sistemática de los Objetivos de desarrollo sostenible.

\section{RESULTADOS Y DISCUSIÓN}

El proyecto educativo pragmático ambiental de reúso de los Neumáticos usados con la ejecución de su proceso fue aplicado en tres unidades educativas de la ciudad de Guayaquil incluyendo a estudiantes con escolaridad inconclusa, contando con 73 docentes que participaron en calidad de guías de los grupos de trabajo establecidos en cada unidad educativa.

Cabe mencionar que se trabajó con un nivel educativo medio correspondiente a décimo curso, quienes abordan temas de los cinco bloques curriculares de la asignatura de Ciencias Naturales relacionando: la tierra un planeta con vida, el suelo y contaminantes sólidos, el reciclaje y reúso.

En la Tabla 1, se muestran los resultados a partir del listado de alternativas planteadas durante la ejecución de actividades propuestas durante la ejecución del PEI TINI en las unidades educativas pertenecientes al Distrito Pascuales. Las alternativas desarrolladas para el reúso de los NFU fueron aplicadas en una muestra de 03 unidades educativas de nivel medio en los estudiantes de octavo, noveno y décimo año que corresponden a educación básica superior, y desarrollaron el Proyecto
Educativo Integral TINI dentro del bloque curricular de la asignatura de Ciencias Naturales.

El $89 \%$ de los docentes consideran que es factible incorporar los Neumáticos Fuera de Uso en actividades que mejoran el proceso de aprendizaje. Ver tabla 1 y figura 2 Los estudiantes participantes incorporaron conocimientos de cuidado del entorno y de los valores intrínsecos del Biocentrismo al buscar alternativas de reúso de los NFU, acompañado de actividades pragmáticas alineadas a la disminución de residuos sólidos especiales como es el caso de los NFU presentes en sitios aledaños o en comunidades próximas a las unidades educativas. Los estudiantes participantes en el PEI TINI emplearon un promedio de 20 a 25 NFU en las actividades implementadas por cada unidad educativa del Distrito 0908 Pascuales 2.

Los resultados anteriores aseveran de la relación existente entre el fomento de los valores a través de la reflexión sistemática con autoconciencia incorporando actividades del PEI TINI., así como la estimulación a la resolución de problemas con responsabilidad por parte de los estudiantes participantes vinculado a la práctica ò pragmatismo. Ver figura 3 y tabla 2.

Otra pregunta que resalta en esta encuesta trata sobre las opciones planteadas por los estudiantes participantes en la selección de actividades y sus resultados fueron asociados por los docentes de acuerdo al trabajo colaborativo alcanzado durante el periodo escolar.

En la tabla 3 y figura 4 se evidenció que las alternativas de elaboración de asientos para patios, separadores de áreas verdes y confección de maceteros representaban las mejores opciones de fomento del trabajo colaborativo entre los estudiantes. En particular el $34 \%$ de la muestra consultada coincide que el diseño de asientos para patios implica mayor trabajo colaborativo.

A continuación, se aprecian fotografías tomadas en diferentes unidades educativas que implementaron el PEI- TINI con sus estudiantes del octavo a décimo ver figura 5 . Nótense factores que competen a la creatividad tales como: Selección de formas y tamaños según los espacios disponibles, combinación de colores y funcionalidad del diseño. Factores que fueron inducidos a desarrollar durante las actividades programadas.

El análisis del antes y después de la implementación del proyecto de reúso de neumáticos usados consideró aspectos tales como: la comunicación 
Tabla 1. Resultados de valoración de nivel de desarrollo sobre fomento de la competencia de reflexión sistémica con autoconciencia ambiental y aprendizaje significativo por uso de NFU en el TINI

¿Cree usted que durante la ejecución del Proyecto TINI se podrían incorporar los NFU en actividades que fomenten la autoconciencia del valor de entorno combinándolo con aprendizaje significativo?

\begin{tabular}{ccc}
\hline ALTERNATIVAS & FRECUENCIA & RESULTADO \\
\hline SI & 610 & $89.70 \%$ \\
NO & 70 & $10.30 \%$ \\
TOTAL & 680 & $100.00 \%$ \\
\hline
\end{tabular}

Fuente: Propia, encuestas en unidades educativas. Distrito 09008, Pascuales 22017

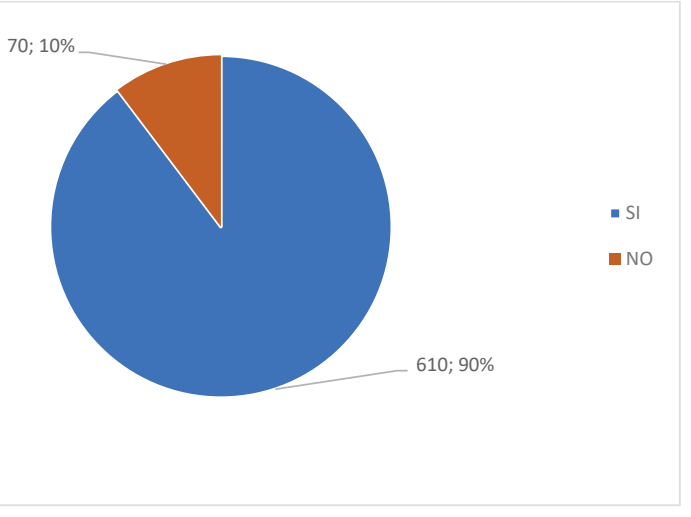

Figura 2. Resultados sobre uso de NFU en proyecto TINI fomento de autoconciencia del valor del entorno combinado con proceso significativo Fuente: Propia, encuestas en unidades educativas. Distrito 09008, Pascuales 22017

Tabla 2. Resultados de valoración de vinculación del pragmatismo ambiental y la competencia de toma de decisión con responsabilidad en actividades del PEI TINI

¿Cree usted que durante la ejecución del Proyecto TINI se alcanza el Nivel de desarrollo cognitivo en resolución de problemas con responsabilidad en la toma de decisiones, a través de: ¿las prácticas de selección, recolección de NFU y elaboración de diseños creativos decorativos como alternativas de reúso?

\begin{tabular}{ccc}
\hline ALTERNATIVAS & FRECUENCIA & RESULTADO \\
\hline SI & 604 & $89 \%$ \\
NO & 76 & $11 \%$ \\
TOTAL & 680 & $100.00 \%$ \\
\hline
\end{tabular}

Fuente: Propia, encuestas en unidades educativas. Distrito 09008, Pascuales 22017

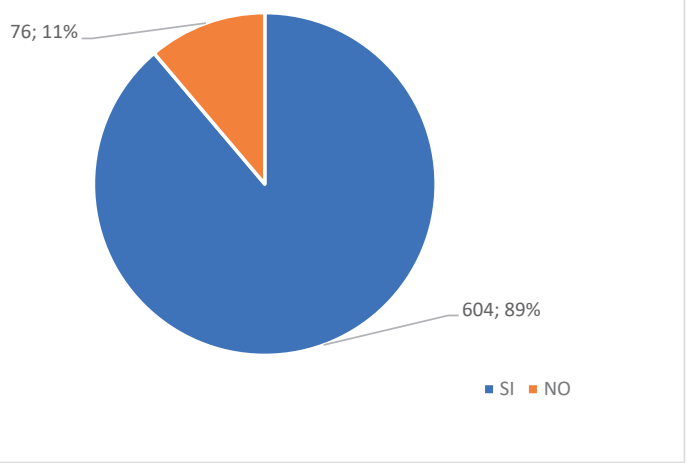

Figura 3 . Vinculación pragmatismo ambiental y competencia en la toma de decisiones con responsabilidad para la resolución de problemas en PEI -TINI Fuente: Propia, encuestas en unidades educativas. Distrito 09008, Pascuales 22017 
Tabla 3. Resultados sobre selección de alternativas de reúso de NFU y la competencia de los ODS -trabajo colaborativo

\begin{tabular}{lc}
\hline \multicolumn{2}{c}{ ¿Cuál de las alternativas planteadas en la matriz de selección de reúso de los NFU y actividades del Proyecto TINI fomentaron el } \\
trabajo colaborativo? & FRECUENCIA \\
\hline \multicolumn{1}{c}{ ALTERNATIVAS } & 106 \\
\hline Confección de maceteros & 78 \\
Elaboración de figuras decorativas & 37 \\
Pisos para gimnasia & 226 \\
Asientos para patio & 44 \\
Columpios & 189 \\
Separadores de Áreas Verdes & 4
\end{tabular}

Fuente: Propia, encuestas en unidades educativas. Distrito 09008, Pascuales 22017

Frecuencias de Selección de Alternativas de reuso NFU

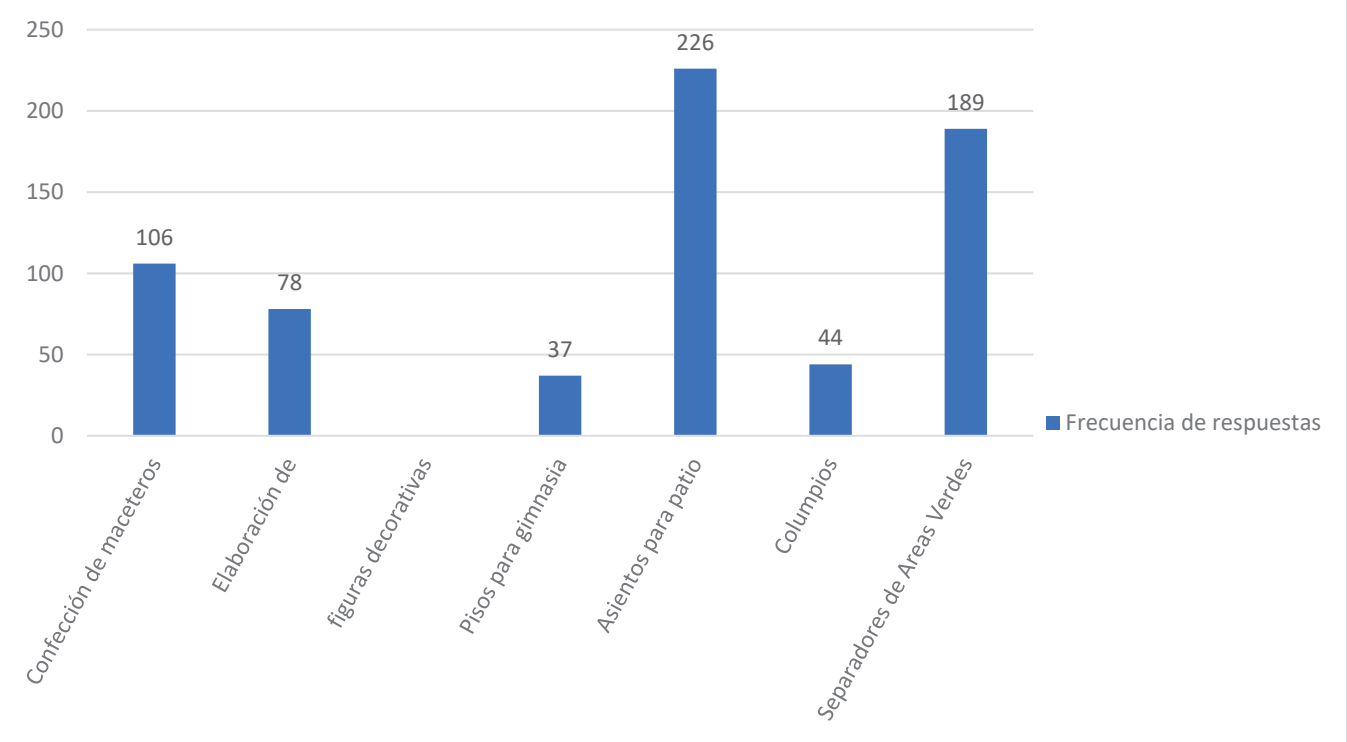

Figura 4. Resultados sobre alternativas de uso de los NFU en proyecto TINI durante el proceso significativo Fuente: Propia, encuestas en unidades educativas. Distrito 09008, Pascuales 22017
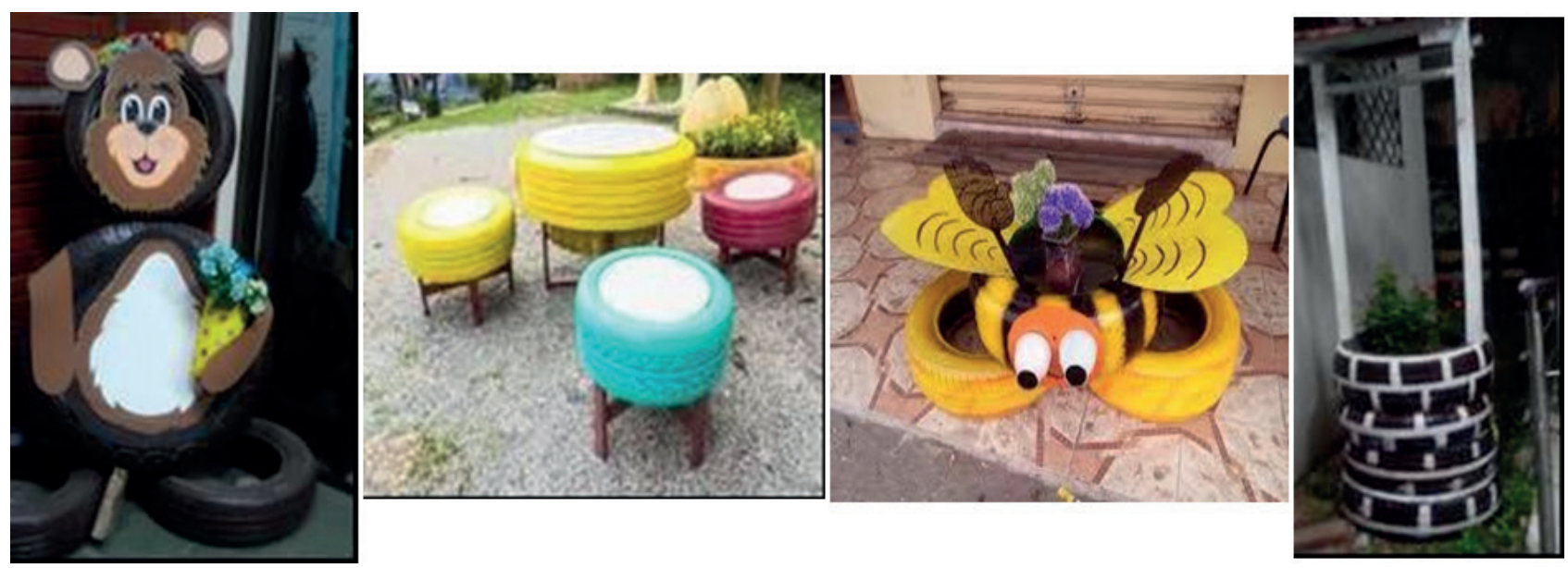

Figura 5. Alternativas del reúso de los NFU en las unidades educativas según matriz de selección de alternativas. Figuras decorativas(a) Asientos (b) Separadores para jardinerìa (c) Bases para maceteros (d)

Fuente: Propia, elaborada por autores 
permanente entre grupos de trabajo para la selección de las alternativas aplicadas, tareas planificadas por fecha límite de acuerdo con los logros del Proyecto TINI y la autoconciencia del cuidado del entorno.

\section{CONTRASTE DE PRUEBA DE HIPOTESIS}

Para el contraste de prueba de hipótesis se estableció lo siguiente:

$\mathrm{H}_{\mathrm{o}=}$ No es posible agrupar las actividades del Reúso pragmático de neumáticos en tres competencias de desarrollo sostenible: análisis crítico, reflexión sistemática y toma de decisión con responsabilidad.

$\mathrm{H}_{1}$ :Es posible agrupar las actividades del Reúso pragmático de neumáticos en tres competencias de desarrollo sostenible: análisis crítico, reflexión sistemática y toma de decisión con responsabilidad.

Decidiéndose en base al tratamiento estadístico de datos, que resultando el valor de $p$ menor que 0,01 se rechaza $\mathrm{H}_{0}$.

Y como conclusión: Es posible agrupar las actividades del Reúso pragmático de neumáticos en tres competencias de desarrollo sostenible, proviniendo de una población con una distribución normal a un nivel de significancia de 0,01. Véase Tabla 4.

El estadístico de chi-cuadrada general es 0,6468 y tiene un valor $p$ de 0.0085 . Puesto que el valor $p$ es menor que el nivel de significancia de 0.01 , el investigador puede rechazar la hipótesis nula. Por lo tanto se concluye que no existe una diferencia significativa y si es posible agrupar las actividades del Reúso pragmático de neumáticos en tres competencias de desarrollo sostenible, las cuales son: análisis crítico, reflexión sistemática y toma de decisión con responsabilidad.

\section{Análisis factorial exploratorio}

El contraste anterior se complementa con el análisis factorial exploratorio y se observa en Tabla 5 que el coeficiente $\mathrm{KMO}$ es igual a 0.678 , que al estar próximo a 1 , es un buen indicador, indica que se puede implementar una reducción dimensional.

Adicionalmente, la prueba de Bartlett es significativa $(P<0,01)$, lo que permite rechazar la hipótesis nula de que la matriz de correlaciones es igual a la matriz identidad, por lo que se concluye que efectivamente existe asociación entre las variables originales y una reducción dimensional es procedente y tiene sentido.

La matriz de componentes estuvo compuesta por:

- Capacidad de formación de grupos para observaciones del entorno afectado por NFU

- Conformación de grupos para recolección de NFU

- Interacciones entre autoridades, docentes y padres de comunidad durante propuestas

- Tipos de NFU encontrados en entorno.

Tabla 4.

\begin{tabular}{|l|c|c|c|}
\hline \multicolumn{4}{|c|}{ Pruebas de chi-cuadrado } \\
\hline & Valor & gl & Sig. asintótica (2 caras) \\
\hline Chi-cuadrado de Pearson & $0,6468^{\text {a }}$ & 11 & 0,0085 \\
\hline Razón de verosimilitud & 26,516 & 11 & 1 \\
\hline N de casos válidos & 680 & & \\
\hline
\end{tabular}

Frecuencia esperada menor que 5. El recuento mínimo esperado es ,02.

Fuente: Propia, elaborada por autores 2019

Tabla 5 .

\begin{tabular}{|lcc|}
\hline \multicolumn{3}{|c|}{ Prueba de KMO y Bartlett } \\
\hline Medida Kaiser-Meyer-Olkin de adecuación de muestreo & 0,678 \\
Prueba de esfericidad de Bartlett & Aprox. Chi-cuadrado & 4308,766 \\
\cline { 2 - 3 } & Sig. & 1485 \\
\hline
\end{tabular}

Nota: valor $p<0,01$ en Objetivos de Desarrollo Sostenible

Fuente: Propia, elaborada por autores 2019 
- Incremento de interacciones personales

- Conformación de subgrupos supervisados por docentes

- Propuestas de diseños tipo reúso

- Funcionalidad de diseños propuestos.

- Comparación entre opciones seleccionadas de diseño decorativo con el ambiente

- Integración de conceptos de seguridad

- Valoración del entorno

- Interacciones para desarrollo del proceso de limpieza de los NFU

- Cumplimiento de metas en actividades asignadas a cada grupo.

- Cumplimiento de tiempos respuesta para elaboración de diseños

En la Figura 6, muestra el "gráfico de codo" indicando el número adecuado de componentes principales entre 3 ó 4 . El análisis factorial exploratorio se efectuó con el paquete estadístico SPSS versión 22. Inicialmente se especificó como método de extracción: "Componentes Principales" o competencias principales tales como: Análisis Crítico, Toma de decisiones con responsabilidad y Reflexión Sistemática.
Se seleccionó una solución factorial sin rotar y la matriz de correlaciones de las muestras tomadas ANTES y DESPUÉS de la Implementación del proyecto pragmático de reúso de NFU con las actividades de las diferentes fases

Para ser más exhaustivo en el análisis se aplicaron 3 tipos de rotaciones: varimax, quartimax y equamax. Ver figura 7 El método de rotación que presentó resultados más claros fue el de varimax. Los residuos se calcularon entre las correlaciones observadas y reproducidas con valores absolutos mayores que 0,05 , en cada uno de los ejes latentes, se consideraron como puntuaciones altas de las variables originales, tanto en la corrida sin rotación como en aquellas que tenían rotaciones.

Las marcas en los ejes biplot $\left(^{*}\right)$ y $(\rightarrow)$ identifican los percentiles 50 y 75 respectivamente. Los marcadores fila CLUSTER1, CLUSTER2 y CLUSTER3, de la muestra original se representan con (+), y los de la muestra de validación se representan con $\left(^{*}\right)$.

Los resultados de la nueva extracción de componentes y la lectura de los reactivos evidenciaron que la nueva agrupación de ítems resultaba más acorde y simplificada, debido a que las competencias de los Objetivos de Desarrollo Sostenible se

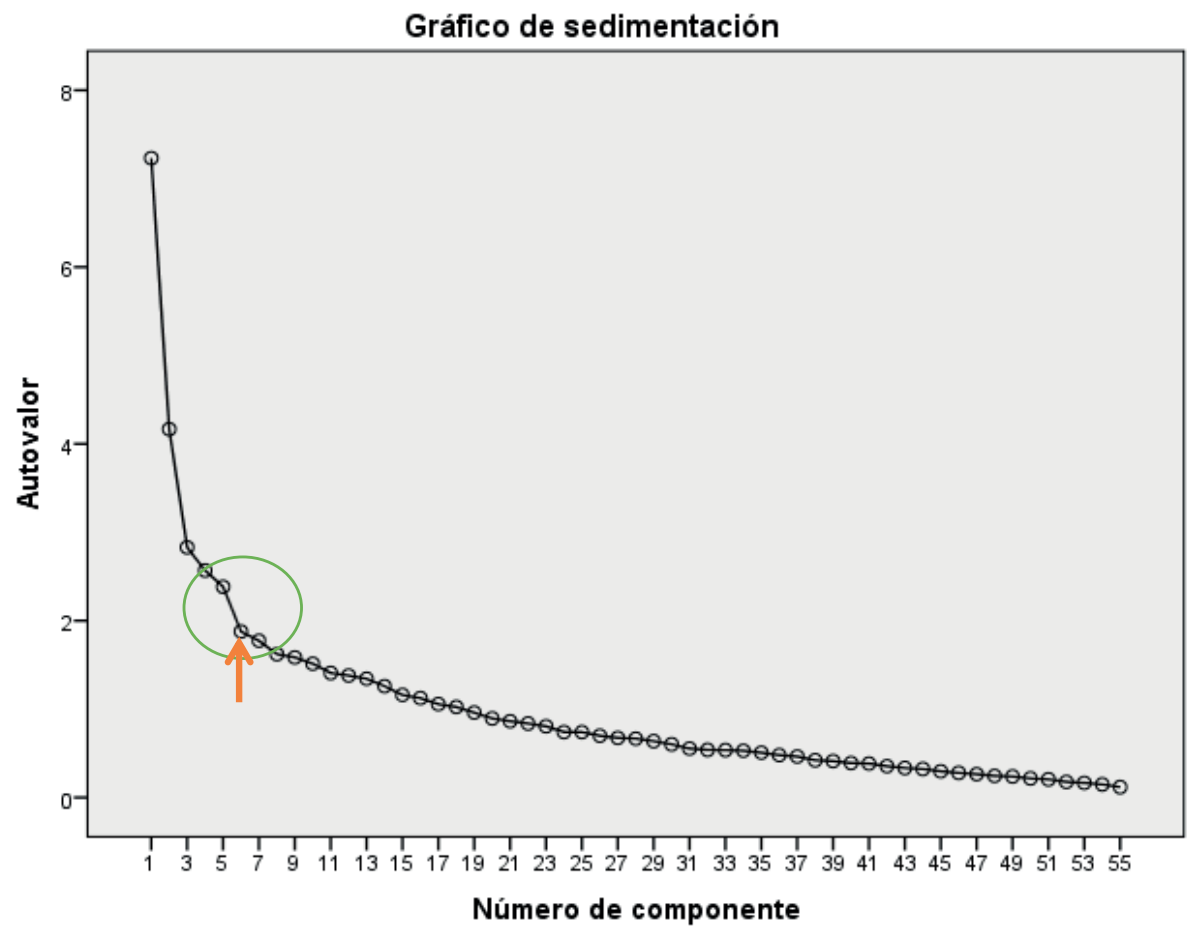

Figura 6. Presentación del Gráfico del Codo

Fuente: Propia, elaborada por autores 2019 


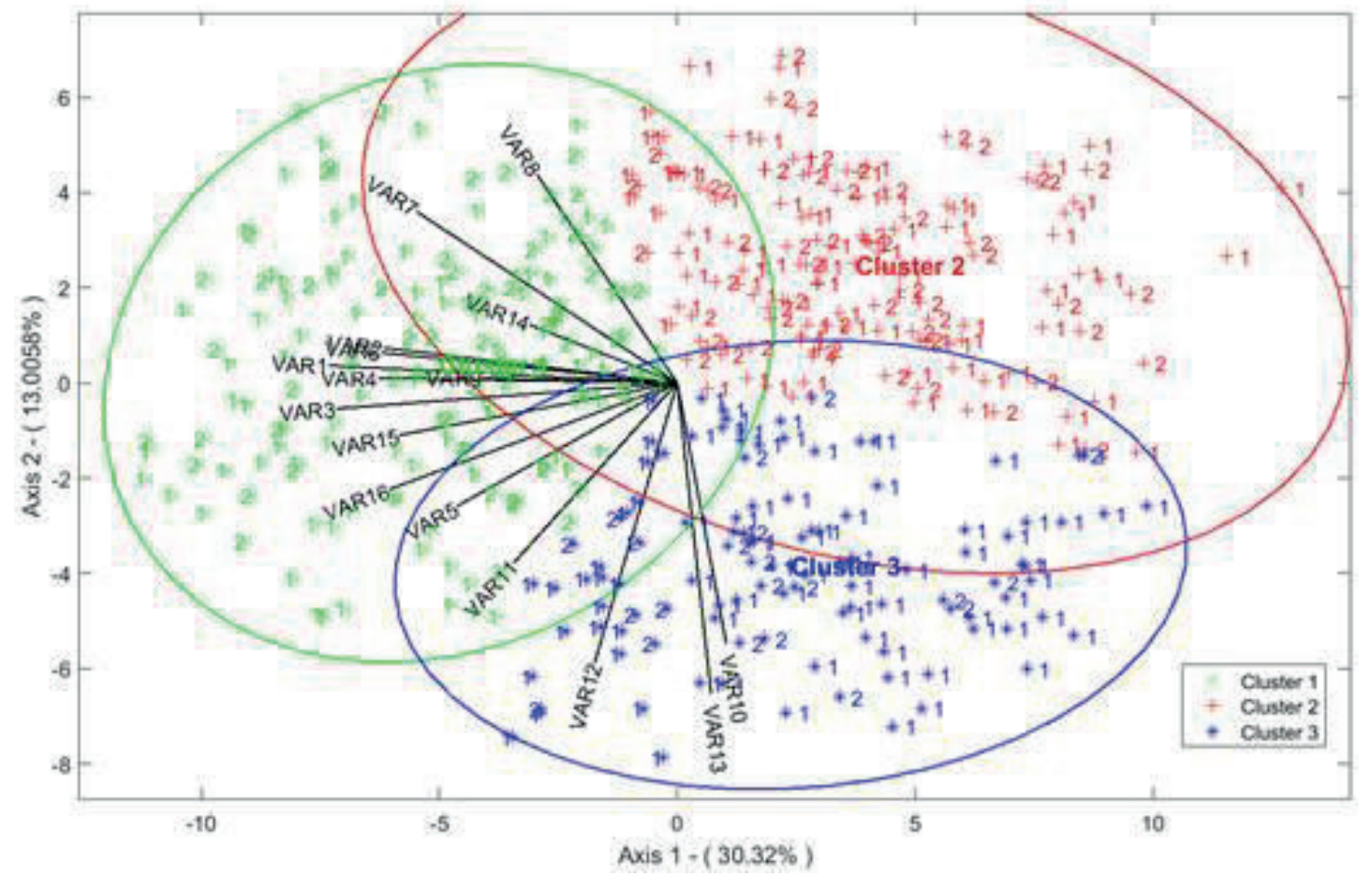

Figura 7. Se muestra el Grafico HJ-BIPLOT

Fuente: Propia, elaborada por autores 2019

deben analizar bajo niveles de desarrollo empleando la observación, tal como se demostró en los resultados arriba detallados, en un espacio de baja dimensión.

Se observa claramente la diferenciación entre los tres tipos de clúster. La probabilidad de presencia de cada variable, se puede aproximar proyectando los marcadores fila sobre cada eje biplot, por lo que, con el fin de facilitar la predicción, se introducen marcas para los percentiles 50 y 75 . Se obtiene un buen ajuste para todas las variables, siendo el porcentaje de bien clasificados superior al $75 \%$, por lo que la predicción de presencia-ausencia de cada variable por cada grupo o clúster es bastante aproximada.

Por lo tanto, se logra implementar una reducción dimensional. La tabla 6 BIPLOT: Principal Normalización (Baricentric Scaling) reforzando la idea de que las actividades del proyecto educativo de reusó pragmático de neumáticos usados pueden agruparse según las competencias de desarrollo sostenible: análisis crítico, toma de decisiones responsables para la resolución de problemas y reflexión sistémica con autoconciencia.
Tabla 6. BIPLOT

Estimation Method: Singular Value Decomposition Type of Biplot: Principal Normalization (Baricentric Scaling) Eigenvalues \& variance explained Inertia

\begin{tabular}{|c|r|r|r|}
\hline Axis & Eigenvalue & Expl. Var. & Cummulative \\
\hline Axis 1 & 2.018 .098 & 30.32 & 30.32 \\
\hline Axis 2 & 865.666 & 13.006 & 43.326 \\
\hline Axis 3 & 602.711 & 9.055 & 52.381 \\
\hline Axis 4 & 423.129 & 6.357 & 58.738 \\
\hline Axis 5 & 358.857 & 5.391 & 64.13 \\
\hline Axis 6 & 305.087 & 4.584 & 68.713 \\
\hline Axis 7 & 297.142 & 4.464 & 73.177 \\
\hline Axis 8 & 266.015 & 3.997 & 77.174 \\
\hline Axis 9 & 255.579 & 3.84 & 81.014 \\
\hline Axis 10 & 236.304 & 3.55 & 84.564 \\
\hline Axis 11 & 220.301 & 3.31 & 87.874 \\
\hline Axis 12 & 195.388 & 2.936 & 90.809 \\
\hline Axis 13 & 183.385 & 2.755 & 93.565 \\
\hline Axis 14 & 163.52 & 2.457 & 96.021 \\
\hline Axis 15 & 149.568 & 2.247 & 98.268 \\
\hline Axis 16 & 115.25 & 1.732 & 100 \\
\hline
\end{tabular}

Fuente: Propia, elaborada por autores 2019 
Para las muestras relacionadas se realiza un análisis comparativo de medias en las muestras tomadas ANTES y DESPUÉS.

La estadística inferencial clásica, y la que considera la asignación de roles asimétricos a las variables (dependientes e independientes), por lo que constituyen áreas abiertas a la investigación, la utilización en las aproximaciones Biplot de técnicas de re-muestreo para el análisis de la estabilidad en las estimaciones y determinación de distribuciones asintóticas de los estimadores, y la inclusión de relaciones de dependencia funcional entre las variables.

\section{CONCLUSIONES}

EI Proyecto Educativo Integral PEI Tierra de niñas, niños y jóvenes para el Buen Vivir TINI, contribuye al fortalecimiento del aprendizaje significativo basado en los pilares del pragmatismo, creatividad y trabajo colaborativo, en las Unidades Educativas participantes: Alonso de Illescas, Adalberto Ortiz y PCEI Eugenio Espejo ubicados en el Distrito 09008, ubicado en Pascuales 2 de la Ciudad de Guayaquil en la Provincia del Guayas.

El listado de selección de alternativas para el reúso de los NFU encontrados en comunidades aledañas a las unidades educativas evidencia durante su estructuración el ajuste conceptual del reúso según la necesidad local de las comunidades del sector de Pascuales 2 aquejadas por la presencia de desechos sólidos especiales tales como los NFU, que generan: trastornos paisajísticos, proliferación de vectores infecciosos, acumulación de desechos en sectores baldíos. Durante la elaboración de la matriz de selección los docentes identificaron factores predominantes como: Selección de formas y tamaños según los espacios disponibles, combinación de colores y funcionalidad de los diseños.

EI PEI TINI alcanzó a reusar un promedio de 118 NFU en las actividades implementadas por 03 unidades educativas durante un periodo escolar anual, favoreciendo el manejo apropiado de estos desechos especiales en el Distrito 09008, ubicado en Pascuales 2 de la Ciudad de Guayaquil en la Provincia del Guayas; así como se logró alcanzar el nivel de desarrollo en las competencias de ODS a través de las actividades pragmáticas ambientales para los estudiantes participantes fomentando el trabajo colaborativo entre grupos de estudiantes destinados a: recolección, diseño, corte y decorado de NFU asistidos por docentes y padres de familia. Esto a su vez permite despertar la reflexión sistemática con autoconciencia de las acciones que pueden programarse durante clases de asignaturas afines.
De la encuesta se desprende que el $34 \%$ de la muestra consultada de docentes coinciden que el diseño de asientos para patios denotó mayor trabajo colaborativo, seguido del $28 \%$ que consideraron a la actividad de elaboración de separadores de áreas verdes.

Se recomienda para posteriores etapas del proyecto establecer un modelo de gestión integral de desechos sólidos para las comunidades del Distrito 09008, ubicado en Pascuales 2 de la Ciudad de Guayaquil en la Provincia del Guayas y se enfatice la separación y aprovechamiento otros desechos como los orgánicos.

El desarrollo de las competencias de reflexión con autoconciencia, y toma de decisiones con responsabilidad para la resolución de problemas, generaron una importante sinergia para visualizar efectos positivos como: incremento de colaboración entre grupos de trabajo estudiantiles, selección de estrategia con alternativas creativas para el mejoramiento del entorno de las unidades educativas participantes durante el Proyecto Educativo de Reúso Pragmático de neumáticos usados, así como la creación de nuevos grupos de trabajo que se interesan en la réplica del proyecto en otros sectores de la ciudad de Guayaquil.

Con respecto al estadístico de la prueba chi-cuadrada general $0,6468^{a}$ cuyo valor $p$ de 0.0085 , se puede notar que el valor $p$ es menor que el nivel de significancia de 0.01 y el investigador puede rechazar la hipótesis nula. Por otro lado, la aplicación de la prueba de Bartlett es significativa $(P<0,01)$, lo que permite rechazar la hipótesis nula debido a que la matriz de correlaciones es igual a la matriz identidad, por lo que se concluye que efectivamente existe asociación entre las variables originales y una reducción dimensional de las actividades del proyecto educativo de reúso pragmático de neumáticos usados es procedente. Estas actividades se agruparon en tres competencias: Análisis Reflexivo con autoconciencia, Toma de decisiones con responsabilidad para resolución de problemas y Reflexión sistémica.

Las actividades de la fase 1: Análisis de Problemática y Formación de los equipos de trabajo tributaron a la competencia ODS de análisis crítico, mientras que las actividades de fase 4: Selección de diseños decorativos se agruparon en la competencia de Toma de decisiones con responsabilidad para la resolución de problemas; y las actividades de la fase 5: Evaluaciones grupales se relacionaron a la Reflexión sistemática y autoconciencia. 
Los estudiantes desarrollaron juicios de valor al entorno durante las actividades, debido a la participación activa en la selección de alternativas de reúso de los NFU dando lugar a la aplicabilidad de los conceptos de sostenibilidad combinado el pragmatismo, despertando el interés a la comunidad por aprender nuevas formas de mejoramiento del entorno bajo esquemas colaborativos; compartiendo experiencias durante varias etapas y su posterior racionalización.

\section{REFERENCIAS}

[1] Bambrick, H. (2011). Climate Change and Health in the Urban Environment: Adaptation Opportunities in Australian Cities Asia- Pacific Journal of Public Health, 23(2), pp.67-79. Recuperado de https://doi. org/10.1177/1010539510391774

[2] Capon, A., Synnott, E., y Holliday, S. (2009). Banism, climate change and health: systems approaches to governance. New South Wales Public Health Bulletin. Recuperado de: https:// www.researchgate.net/publication/24177269_ Urbanism_climate_change_and_health_ systems_approaches_to_governance

[3] Collazos, C., y Mendoza, J. (2006). Cómo aprovechar el "aprendizaje colaborativo" en el aula. Educación y Educadores, pp. 6176. Recuperado de https://www.redalyc.org/ pdf/834/83490204.pdf

[4] Cozar, J. (2004). Ecopragmatismo: protección ambiental y calidad de la democracia. Universidad de la Laguna, Congreso de Sociedad de Lógica, Metodología y Filosofía de la Ciencias, España, pp. 52-56. Recuperado de: https://dialnet.unirioja.es/servlet/ articulo?codigo $=4398238$

[5] Dourojeanni, A. (2000). Manual CEPAL ECLAC Procedimientos de gestión para el desarrollo sustentable, Serie Manuales, Impreso en Naciones Unidas, Santiago de Chile, pp. 131147. Recuperado de https://repositorio.cepal. org/handle/11362/5564

[6] Flavell, J. (1979). Metacognition and cognitive monitoring: A new area of cognitivedevelopmental inquirí. American Psychology. 34(10), 906-911. Recuperado de: https:// psycnet.apa.org/record/1980-09388-001

[7] Gualdrón, J. M. (2007). El reciclaje, la forma más fácil de mantener nuestro planeta vivo. Recuperado de: https://www.gestiopolis.com/ reciclaje-forma-mas-facil-mantener-nuestroplaneta-vivo/
[8] Institución Educativa Unidad de Formación Artesanal Fiscal "Eugenio Espejo" (2015) Código de Convivencia.

[9] Instituto Nacional de Estadísticas - INEC (2015). Reporte Ecuador en cifras 2015. Recuperado de https://www.ecuadorencifras. gob.ec/estadisticas-de-transporte-2015/

[10] Instituto Latinoamericano y del Caribe de Planificación Económica y Social (2000). Material docente sobre gestión y control de proyecto. Recuperado de https://repositorio. cepal.org/bitstream/handle/11362/5563/1/ S2000925_es.pdf

[11] Joas, H. (1998). El pragmatismo y la teoría social. Centro de Investigaciones Sociológicas (CIS). Revista Política y Sociedad de la Universidad Pública de Navarra, pp.177-187. Recuperado de https://core.ac.uk/download/ pdf/38819189.pdf

[12] Murillo, Y. (2004). La perspectiva antropológica en Filosofía de la Ciencia. Actas del IV Congreso de la Sociedad de Lógica, Metodología y Filosofía de la Ciencia en España, Valladolid, pp. 203-207. Recuperado de: https://dialnet. unirioja.es/servlet/articulo?codigo $=4398683$

[13] Ramírez, A., Sánchez, J., y García, A. (2003). Ensayo El Desarrollo Sustentable: Interpretación Análisis. Revista del Centro de Investigación. México. 6(21), 57-59. Recuperado de: https://www.redalyc.org/ pdf/342/34202107.pdf

[14] Rolston III, H. (2004). Ética ambiental: valores en el mundo natural y deberes para con él. Revista Dialnet. Recuperado de: https://dialnet. unirioja.es/servlet/articulo?codigo $=2596942$

[15] Salazar, V. (2012). La visión crítica del pragmatismo ambiental respecto a la ética ambiental tradicional. Euphyía - Revista de Filosofía. Recuperado de: https://www. researchgate.net/publication/332336329_La_ vision_critica_del_pragmatismo_ambiental_ respecto_a_la_etica_ambiental_tradicional

[16] Tünnermann, C., y Souza, M. (2003). Desafíos de la Universidad en la Sociedad del Conocimiento Cinco Años Después de la Conferencia Mundial sobre Educación Superior. Unesco Recuperado de: http://www. unachi.ac.pa/assets/descargas/planificacion/ doc-8-desafios_la_universidad.pdf

[17] Weston, A. (1985). Beyond Intrinsic Value: Pragmatism in Environmental Ethics. Environmental Ethics 7(4), 321-339. Recuperado de: https://philpapers.org/rec/WESBIV-2 